\title{
Thioredoxin 1 Inactivation Mediates the Impairment of Ischemia-Induced Angiogenesis and Further Injury in Diabetic Myocardium
}

\author{
Rongrong Hou ${ }^{a, b}$ Mingzhi Shen ${ }^{a, c}$ Rutao Wang ${ }^{a}$ Haitao Liu $^{a}$ Chao Gao $^{a}$ \\ Jing $\mathrm{Xu}^{\mathrm{b}}$ Ling Tao ${ }^{\mathrm{a}}$ Zhiyong Yin ${ }^{\mathrm{a}}$ Tao Yin $^{\mathrm{a}}$ \\ a Department of Cardiology, Xijing Hospital, the Fourth Military Medical University, Xi'an, PR China; \\ ${ }^{b}$ Department of Endocrinology, the Second Affiliated Hospital, Xi'an Jiaotong University, Xi'an, PR China; \\ 'Department of Cardiology and National Clinical Research Center of Geriatrics Disease, Hainan Hospital \\ of PLA General Hospital, Sanya, PR China
}

\section{Keywords}

Thioredoxin · Angiogenesis · Diabetes · Myocardial infarction · Endothelial cell

\begin{abstract}
Diabetes mellitus (DM)-induced impairment of collateral formation has been demonstrated in subjects with coronary artery disease, which contributes to unfavorable prognosis among diabetic individuals. In our previous studies, thioredoxin 1 (Trx1) activity was shown to be decreased in diabetic cardiac tissues, but the reason of Trx1 inactivation and whether it mediates the impaired angiogenesis in ischemic myocardium is still to be identified. As thioredoxin-interacting protein (TXNIP), an endogenous inhibitor of Trx, is overexpressed in DM due to carbohydrate response element within its promoter, we hypothesized that inhibition of Trx1 by enhanced TXNIP expression in endothelial cells may play a role in hyperglycemia-induced impairment of angiogenesis. In the present study, we found that high glucose-mediated increase of TXNIP expression and TXNIP-Trx1 interaction induced the impairment in endothelial cell function and survival, since these detrimental effects are rescued by silencing TXNIP with small interfering RNA. In diabetic mice,
\end{abstract}

TXNIP knockdown or recombinant human Trx1 treatment counteracted the impairment of angiogenesis, alleviated myocardial ischemic injury, and improved survival rate. All these data implicate that TXNIP upregulation and subsequently the increased formation of TXNIP-Trx1 complex is a novel pathologic pathway by which DM induces insufficient angiogenesis and thereby exacerbates myocardial ischemia injury.

c) 2020 S. Karger AG, Basel

\section{Introduction}

Collateral circulation, an important adaptive response for myocardial ischemia, can reduce myocardial ischemia area, decrease the size of myocardial infarction (MI), preserve left ventricular function and viability, and reduce the risk of death. However, pathetically, diabetes mellitus (DM)-induced impairment of collateral formation in ischemic myocardium has been demonstrated in experimental and clinical studies $[1,2]$, which play a critical role

R.H., M.S., and R.W. contributed equally to this work.

\section{KARGER}

(C) 2020 S. Karger AG, Basel

karger@karger.com

www.karger.com/jvr
Dr. Tao Yin or Dr. Zhiyong Yin

Department of Cardiology, Xijing Hospital

The Fourth Military Medical University

Xi'an, Shaanxi 710032 (PR China)

E-Mail yintaofmmu@163.com or zhiyong_yin@163.com 
for the increased mortality and morbidity among coronary artery disease patients. But the mechanism underlying diabetes-related impairment of ischemia-mediated angiogenesis remains to be defined.

Thioredoxin (Trx), apart from its very important antioxidative role, also acts as a transcriptional and growth factor, which is indicated to be a key regulator of cardiovascular homeostasis [3, 4]. In mammalian cells, there are two isoforms of Trx, called Trx1 and Trx2. Trx 1 is mainly located in cytosol but also translocates to the nucleus, whereas Trx 2 is located only in mitochondria. Our previous study has demonstrated that inactivation of Trx1 increases vulnerability of diabetic hearts to ischemia/reperfusion $(I / R)$ injury $[5,6]$. Increasing evidence suggests that Trx1 modulates the process of angiogenesis [7]. Pharmacological or genetic induction of Trx 1 in myocardium can enhance ischemia-mediated angiogenesis [8$10]$.

Trx-interacting protein (TXNIP) is the endogenous inhibitor of Trx, which is strongly induced by glucose due to a carbohydrate response element within its promoter [11]. Overexpression of TXNIP induced by hyperglycemia interacts with Trx1 and inhibits its nuclear translocation, blocking Trx1-dependent gene transcription and influencing the expression of cell death and survival genes [7]. We therefore hypothesized that inhibition of Trx1 by TXNIP exerts a causative effect on impairment of myocardial ischemia-induced angiogenesis under diabetic conditions.

\section{Materials and Methods}

In vivo Experiments

Induction of Diabetes

Male C57 mice at the age of 6-8 weeks were used for the present study. They were randomly grouped into normal and diabetic mice as they received 5 days of daily intraperitoneal injection of vehicle (0.1 M citrate buffer, $\mathrm{pH} 4.5)$ alone or streptozotocin (Sigma, USA) with $50 \mathrm{mg} / \mathrm{kg}$ body weight dissolved in $0.1 \mathrm{M}$ citrate buffer. Blood glucose levels were tested 5 days after streptozotocin injection. Animals with glucose levels $\geq 16.7 \mathrm{mM}$ were considered diabetic mice. The animals were subjected to MI 30 days after the establishment of diabetes. Age-matched male C57 mice were used as comparable controls.

\section{Surgical Procedure}

Mice were anesthetized with $2 \%$ isoflurane, and MI was induced by permanent left anterior descending coronary artery ligation, as described in our previous studies [12]. Adenovirus expressing shTXNIP or the negative control was administered intramyocardially at 4 different sites into the left anterior free wall adjacent to the point of ligation (border zone surrounding the infarct) before the MI surgery. Animals were reanesthetized, the heart tissue was collected after 6 days for measurement of angiogenesis, apoptosis, and proliferation of endothelial cells, and for dihydroethidium staining, immunofluorescence, and Western blot analysis.

Determination of Cardiac Function and Myocardial Fibrosis

Echocardiography (VisualSonicsVeVo 770, Canada) was applied to assess cardiac function 30 days after coronary occlusion. Then the hearts were harvested for analysis of Masson's trichrome staining to assess myocardial fibrosis.

Immunofluorescence for Capillary Density

The hearts were harvested 30 days after MI with or without additional treatments as indicated. The frozen heart sections $(10 \mu \mathrm{m})$ were prepared as described previously and used for capillary density staining and analysis. Briefly, the sections were stained for capillary density using anti-CD31 (1:100 in antibody dilution; Biolegend, USA) followed by incubation with the secondary Fluor-594 antibodies (1:200 in antibody dilution; Earth, USA). The fluorescence was captured by confocal laser scanning microscopy (Nikon, Japan).

Immunofluorescence for Endothelial Cell Expression of Ki67 and CD31 in the Myocardium

The mice were sacrificed 4 days after MI with/out additional treatments as indicated, the hearts were removed, and frozen heart sections $(10 \mu \mathrm{m})$ were prepared as described previously [8]. Immunofluorescence assessment of endothelial cell expression of Ki67 was carried out using endothelial cell specific rat monoclonal anti-CD31 (1:100 in antibody dilution; Biolegend, USA) and rabbit monoclonal anti-Ki67 (1:100 in antibody dilution; Abcam, UK), followed by staining with goat anti-rat secondary Fluor-594 antibody (1:200 in antibody dilution; Earth, USA) and goat anti-rabbit secondary Alexa Fluor 488 (1:200 in antibody dilution; Invitrogen, USA). The sections were observed and images were captured by confocal laser scanning microscopy (Nikon, Japan).

Immunoprecipitation for TXNIP-Trx1 Interaction

Trx1 in homogenized cardiac tissues was immunoprecipitated with a monoclonal antibody against Trx1 (Redox Bioscience). After sample separation, TXNIP-Trx1 interaction was determined by immunoblotting using a primary antibody against TXNIP. Following incubation with horseradish peroxidase-conjugated secondary antibody (Cell Signaling Technology), the blot was developed with an ECL-Plus chemiluminescence reagent kit (Amersham) and visualized with UVP Bio-Imaging Systems. Blot densities were analyzed with VisionWorks LS Acquisition and Analysis Software.

\section{Real Time-PCR}

Total RNA was extracted using TRIzol (Invitrogen, USA). cDNA was synthesized with QuantiTect Reverse Transcription Kit (Takara). Expression analysis was performed on an ABI prism 7500 with the Power SYBR Green PCR Master Mix (Takara). The following primer sequences were used: mouse TXNIP sense 5'-ATGGCCAGACCAAAGTGTTC-3' , antisense 5' -GGCTGTCTTGAGAGTCGTCC-3'; mouse GAPDH sense ${ }^{\prime}$-GGTGAAGGTCGGTGTGAACG-3' , antisense $5^{\prime}$-CTCGCTCCTGGAAGATGGTG-3'. GAPDH was used as endogenous control. 


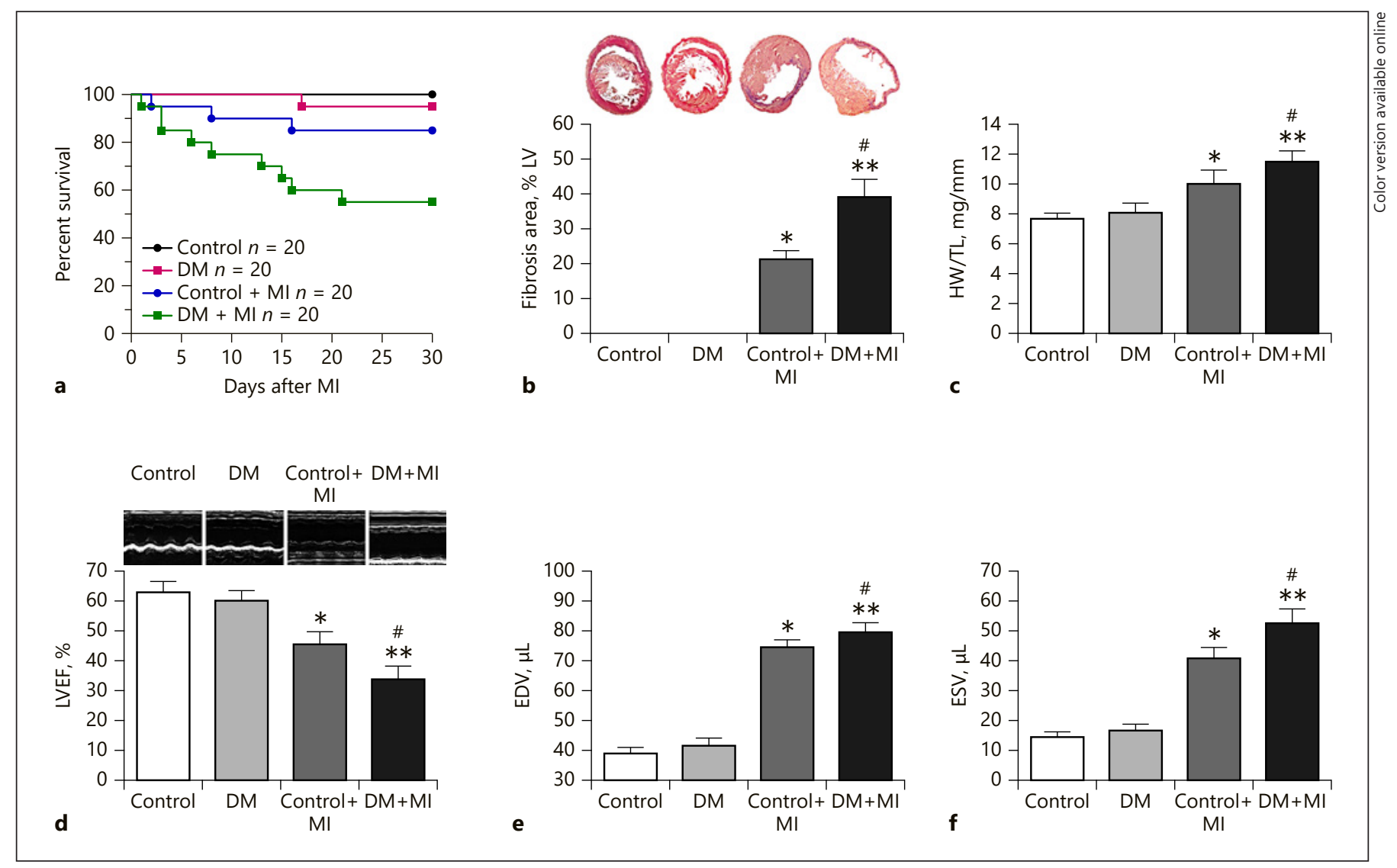

Fig. 1. DM intensified MI-induced myocardial injury $\left(n=6,{ }^{*} p<\right.$ 0.05 vs. control, ${ }^{* *} p<0.05$ vs. DM, ${ }^{\#} p<0.05$ vs. control $\left.+\mathrm{MI}\right)$. a Survival rates of the control and DM mice subjected to sham or MI. Mouse numbers in each group are indicated. b Measurements of myocardial remodeling. Representative pictures of myocardial fibrosis are shown by Masson's trichrome staining in the upper

Western Blot Analysis

The mice were sacrificed at indicated time points and left ventricular risk area sections were harvested. Heart tissue was lysed by RIPA containing a protease inhibitor cocktail (Roche, Germany). Proteins were subjected to SDS-PAGE gels and transferred to nitrocellulose membranes (Millipore, USA). After blocking with 5\% non-fat milk, the membranes were probed overnight at $4{ }^{\circ} \mathrm{C}$ with TXNIP (1:1,000, MBL, Japan), GAPDH (1:5,000, Santa Cruz, USA). Secondary antibodies conjugated to IRDye TM 800 (1: 15,000, Rockland, USA) were detected using an Odyssey infrared imaging system (LI-COR, USA).

Trx1 Activity Assay

Trx1 activity was determined by previously described insulin disulfide reduction assay [6]. In brief, $40 \mu \mathrm{g}$ of cellular protein extract was preincubated with $2 \mu \mathrm{L}$ of activation buffer $(100 \mathrm{mM}$ HEPES, $2 \mathrm{mM}$ EDTA, $1 \mathrm{mg} / \mathrm{mL}$ BSA, and $2 \mathrm{~mm} \mathrm{DTT}$ ) at $37^{\circ} \mathrm{C}$ for $15 \mathrm{~min}$ for Trx 1 reduction. Samples were mixed with $20 \mu \mathrm{L}$ of reaction buffer (100 mM HEPES, 2 mM EDTA, $0.2 \mathrm{mM} \mathrm{NADPH}$, and $140 \mu \mathrm{M}$ insulin). The reaction was initiated by mammalian Trx panel. Quantification of myocardial fibrosis area is indicated in the lower panel. c Ratio of heart weight (HW) to tibia length (TL). d-f Echocardiographic analysis of cardiac function. Representative $M$ mode images are shown in the upper panel in $\mathbf{d}$. Left ventricular ejection fraction (LVEF) value is indicated in the lower panel in $\mathbf{d}$. EDV and ESV values are indicated in $\mathbf{e}$ and $\mathbf{f}$.

reductase $(1 \mu \mathrm{L}, 15 \mathrm{mU}$, Sigma). Samples were incubated for 30 $\min$ at $37^{\circ} \mathrm{C}$. The reaction was terminated by $125 \mu \mathrm{L}$ of stopping solution $(0.2 \mathrm{M}$ Tris- $\mathrm{HCl}, 10 \mathrm{M}$ guanidine- $\mathrm{HCl}$, and $1.7 \mathrm{~mm} 3$-carboxy-4-nitrophenyl disulfide) followed by absorption measurement at $412 \mathrm{~nm}$. Trx1 activity was expressed as oxidized NADPH micromol per minute per milligram $(\mu \mathrm{mol} / \mathrm{min} / \mathrm{mg})$ of protein.

\section{In vitro Experiments}

Cell Culture

Human umbilical vein endothelial cells (HUVECs) were purchased from American Type Culture Collection (ATCC, USA). The cells were cultured in DMEM medium (Hyclone, USA) with antibiotics (Invitrogen, USA) containing 10\% FBS (Gibco, USA) and used between passages 3 and 6 . HUVECs grown under normal glucose $(5.55 \mathrm{mM})$ and high glucose $(33 \mathrm{mM})$ concentrations were used for further treatment as indicated.

RNA Interference

TXNIP-specific short interfering RNA (siTXNIP) was purchased from GenePharma (Shanghai, China). The target sequenc- 


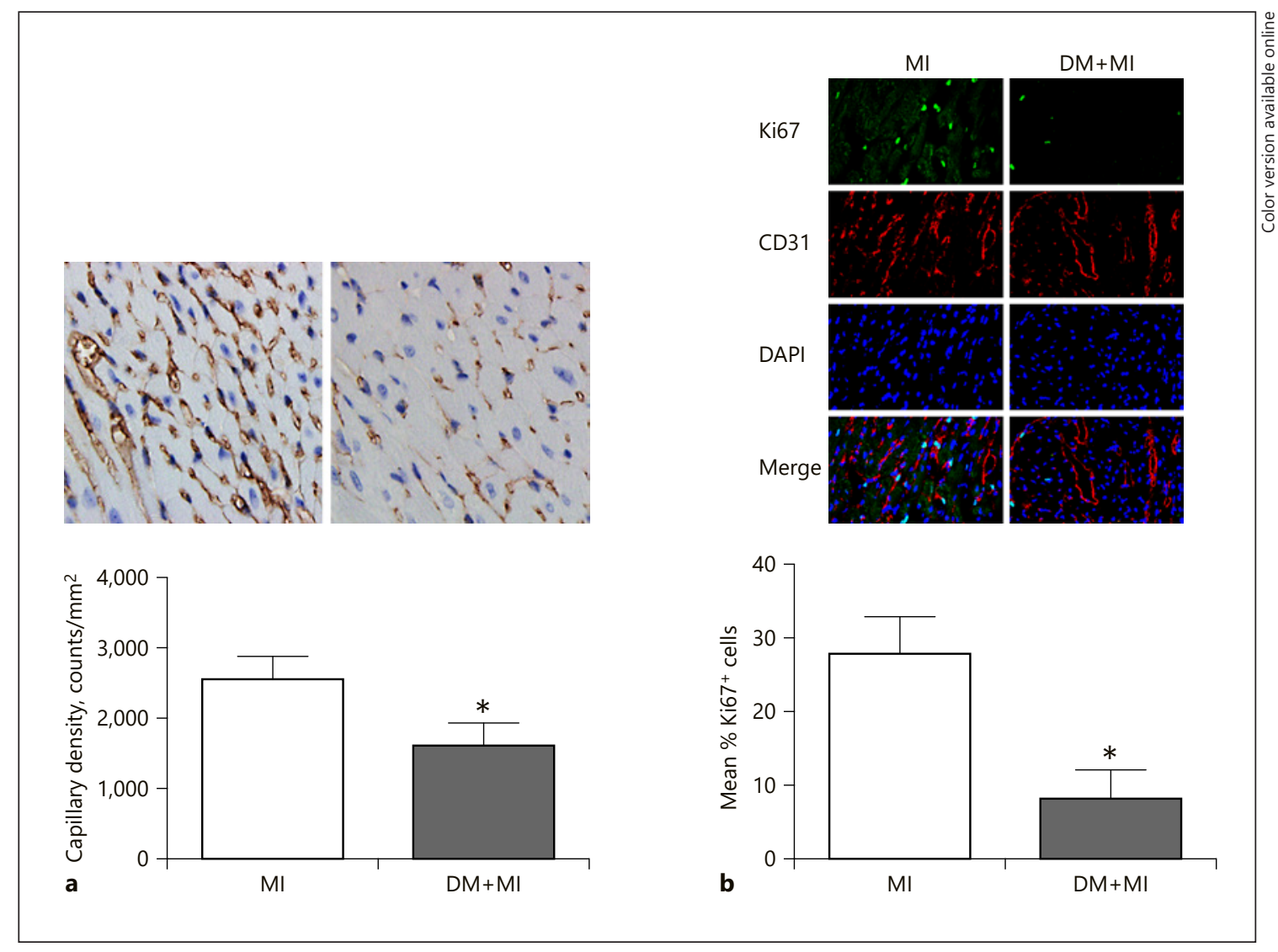

Fig. 2. DM reduced MI-induced angiogenesis $\left(n=6,{ }^{*} p<0.05\right.$ vs. MI). a Vessel density in the infarcted hearts measured by immunohistochemistry staining of CD31. Representative images are shown in the upper panel. Quantitative analysis is indicated in the lower panel. b Proliferation of endothelial cells in myocardium assessed by Ki67 and CD31 double staining. Representative images are shown in the upper panel. Quantitative analysis is indicated in the lower panel.

es of human TXNIP were 5'-AAGCCGTTAGGATCCTGGCTT- $3^{\prime}$, and $5^{\prime}$-AATTCTCCGAACGTGTCACGT- ${ }^{\prime}$ for negative control siRNA. HUVECs were transfected with siRNA by LipofectamineTM 2000 (Invitrogen) according to the manufacturer's instructions. For in vivo endothelial knockdown of TXNIP, oligos encoding shRNAs against TXNIP were cloned into the adenovirus vector, in which the promoter is the 2500-bp VE-Cad promoter, and the flanking sequence is the miR30a structure. Knockdown efficiency was confirmed by Western blot.

\section{TUNEL Assay}

The in situ TUNEL cell death detection kit (Roche, Germany) was used to assess apoptotic cells according to the manufacturer's instructions. Briefly, HUVECs were fixed with $4 \%$ paraformaldehyde and permeabilized in $0.3 \%$ Triton X-100 $(100 \mu \mathrm{L})$ and then washed twice with PBS. DNA breaks were tagged through incubation $\left(1 \mathrm{~h}, 37^{\circ} \mathrm{C}\right)$ with terminal deoxynucleotidyl transferase and nucleotide mixture containing fluorescein isothiocyanate-conjugated dUTP. Cell nuclei were stained with DAPI, the TUNEL-positive and total nuclei were observed, and images were captured under a laser scanning confocal microscopy (Nikon, Japan).

\section{Migration Assay}

Briefly, Transwell insert chambers (Corning, USA) with $8-\mu \mathrm{m}$ pore filters were used. $1 \times 10^{4}$ HUVECs $(200 \mu \mathrm{L})$ were placed in the upper chamber in DMEM medium without fetal bovine serum. The chamber was placed in a 24-well plate containing normal or high glucose DMEM medium (with 10\% FBS) with or without siTXNIP. After incubation for $18 \mathrm{~h}$, the cells on the lower side of filter surface were fixed with $4 \%$ formaldehyde. Migrated cells were visualized by crystal violet staining. Five random high-power microscopic fields (100× magnification) per filter were captured, and the numbers of cells were counted.

\section{Quantification of VEGF}

After treatment, cell culture media was analyzed to determine the concentrations of VEGF using the ELISA kits (R\&D systems).

\section{Statistical Analysis}

All data were expressed as mean \pm SEM and were analyzed using ANOVA, followed by Bonferroni correction for post hoc $t$ test. A value of $p<0.05$ was considered statistically significant. 


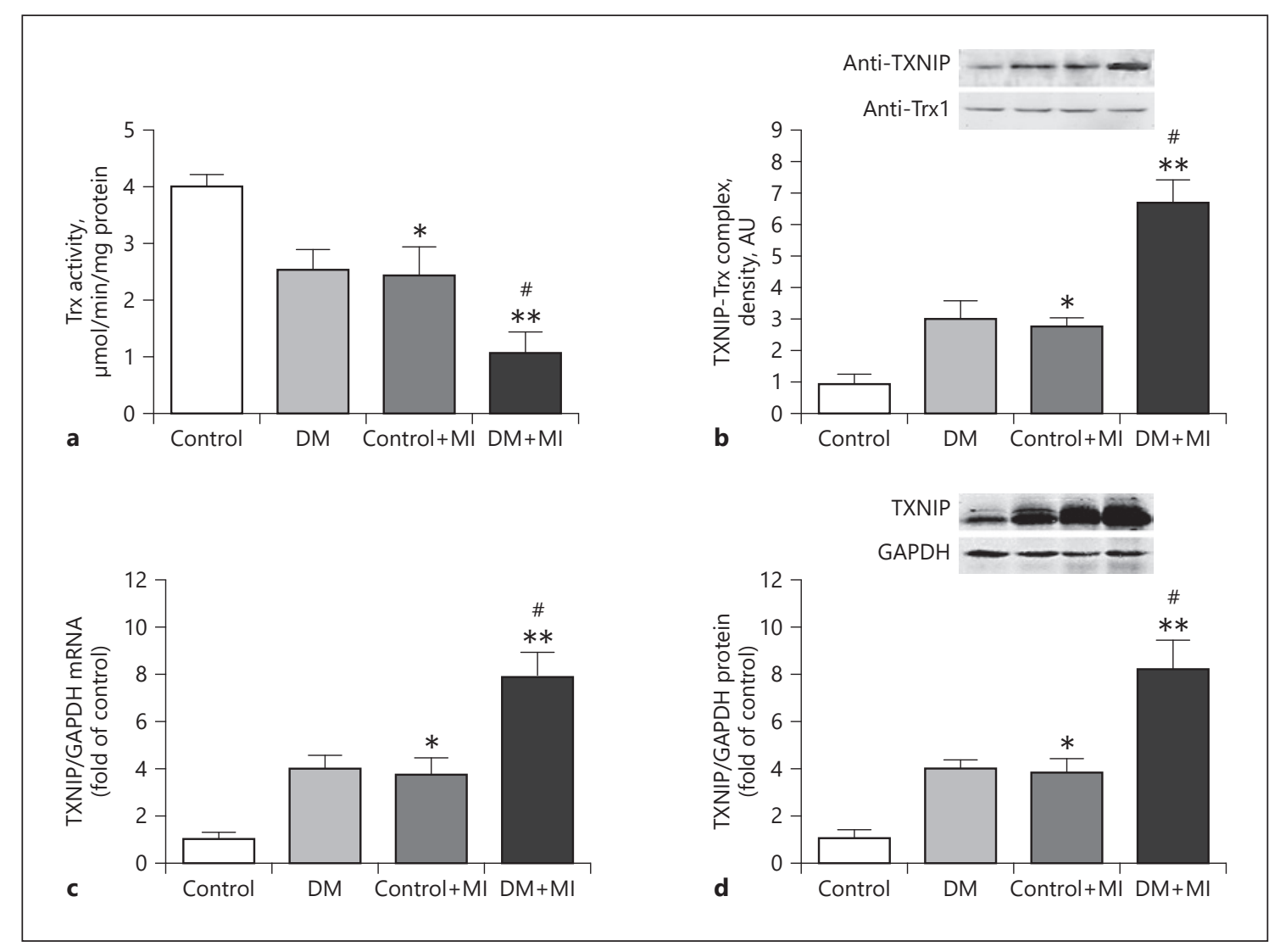

Fig. 3. DM further inhibited Trx1 activity due to increased TXNIP expression and TXNIP-Trx1 interaction in myocardium under MI condition $\left(n=6,{ }^{*} p<0.05\right.$ vs. control, ${ }^{* *} p<0.05$ vs. DM, ${ }^{*} p<0.05$ vs. control $\left.+\mathrm{MI}\right)$. Trx1 activity, TXNIP-Trx1 interaction, and TXNIP mRNA and protein determined by insulin disulfide reduction assay (a), immunoprecipitation (b), RT-PCR (c), and Western blot (d), respectively.

\section{Results}

\section{DM Intensified MI-Induced Myocardial Injury in \\ Relation to Reduced Angiogenesis}

Compared with the control group, post-MI survival rate in the diabetic group was significantly lower. Diabetes increased MI-induced pathological remodeling, as indicated by increased myocardial fibrosis and heart mass (Fig. 1). Consistently, diabetic mice subjected to MI exhibited significantly lower left ventricular ejection fraction than control mice (Fig. 1). Taken together, these data showed that diabetes exacerbated cardiac remodeling after MI, further impaired cardiac contractile function, and reduced the overall survival rate.

As angiogenesis is a compensatory mechanism in response to the injury resulted by MI, the effect of diabetes on myocardial angiogenesis was then assessed. As illustrated in Figure 2, diabetes significantly decreased capillary density and the percentage of Ki67 and CD31 double positive cells in the border zone 30 days after MI. Collectively, it suggested that diabetes significantly reduces endothelial cell proliferation and subsequently angiogenesis, which was a potential underlying mechanism in DMimpaired cardiac repair after MI.

\section{DM Further Inhibited Trx1 Activity due to Increased TXNIP Expression and TXNIP-Trx1 Interaction in Myocardium under MI Condition}

Accumulating evidence supports that Trx1, an antioxidant molecule, has a proangiogenic ability and modulates the angiogenesis process; therefore, it might represent a promising therapeutic target for the ischemic disease $[8,9]$. As shown in Figure 3, Trx 1 activity, determined by insulin disulfide reduction assay, was significantly further decreased in the DM group after MI. Since the activity of Trx1 is mainly regulated through its interaction with TXNIP, the expression of TXNIP and TXNIP-Trx1 interaction were measured. DM intensified the upregula- 


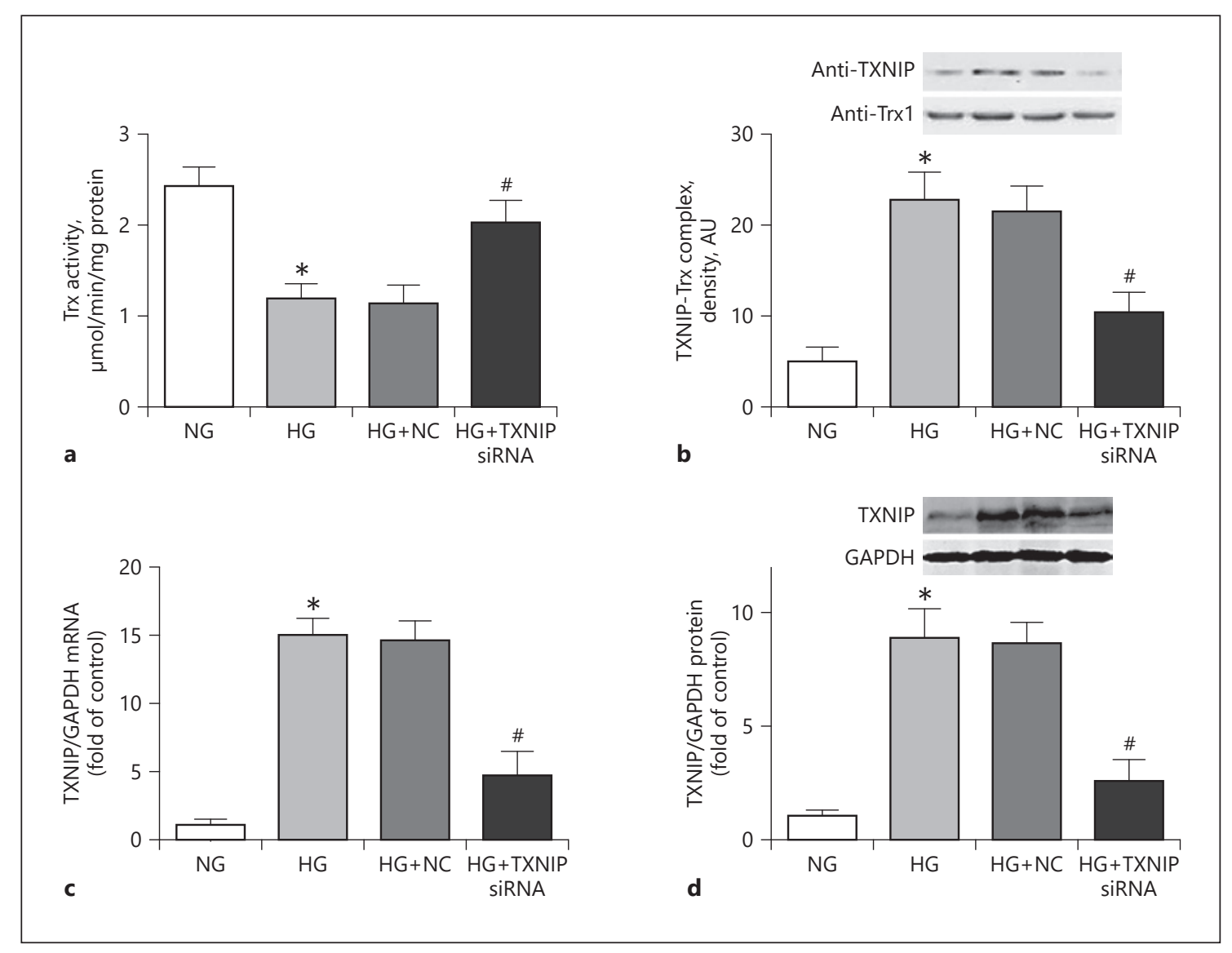

Fig. 4. High glucose inactivated Trx1 through increasing TXNIP expression and TXNIP-Trx1 interaction in HUVECs $\left(n=5,{ }^{*} p<0.05\right.$ vs. NG, ${ }^{\#} p<0.05$ vs. HG + NC). Trx1 activity, TXNIP-Trx1 interaction, and TXNIP mRNA and protein determined by insulin disulfide reduction assay (a), immunoprecipitation (b), RT-PCR (c), and Western blot (d), respectively.

tion of TXNIP expression and thus the TXNIP-Trx1 interaction in mice subjected to MI (Fig. 3). It was reported that hyperglycemia activated TXNIP expression by recruitment of the carbohydrate response element-binding protein (ChREBP) to the TXNIP promoter; thus, DMinduced upregulation of TXNIP reduced Trxl activity through its interaction with Trx1, accounting for the impaired angiogenesis in myocardium.

\section{High Glucose Inactivated Trx1 through Increasing TXNIP Expression and TXNIP-Trx1 Interaction in HUVECS}

Hyperglycemia is a major risk factor in the setting of $\mathrm{DM}$, and endothelial cell plays a key role in ischemia-induced angiogenesis. We hypothesize that TXNIP-Trx1 axis affects angiogenic process through coordinately regulating endothelial cells. In vitro, we detected TXNIP expression, TXNIP-Trx1 interaction, and Trx 1 activity in endothelial cells cultured in high glucose conditions. As illustrated in Figure 4, high glucose increased TXNIP expression, enhanced TXNIP-Trx1 interaction, and decreased Trx1 activity in HUVECs. TXNIP knockdown through TXNIP siRNA decreased the formation of TXNIP-Trx1 complex and partially reversed high glucose-induced Trx1 inactivation in HUVECs (Fig. 4). Therefore, high glucose induced the increase of TXNIP expression, which contributes to the Trx 1 inactivation in endothelial cells.

\section{High Glucose Mediated the Impaired Angiogenic}

Abilities in Endothelial Cells via Trx1 Inactivation

To identify whether the Trx 1 inactivation affected the angiogenic abilities of endothelial cells, the apoptosis, migration, and secretion of endothelial cells were measured in vitro. High glucose increased apoptosis, inhibited migration, and reduced VEGF secretion in HUVECs (Fig. 5). 


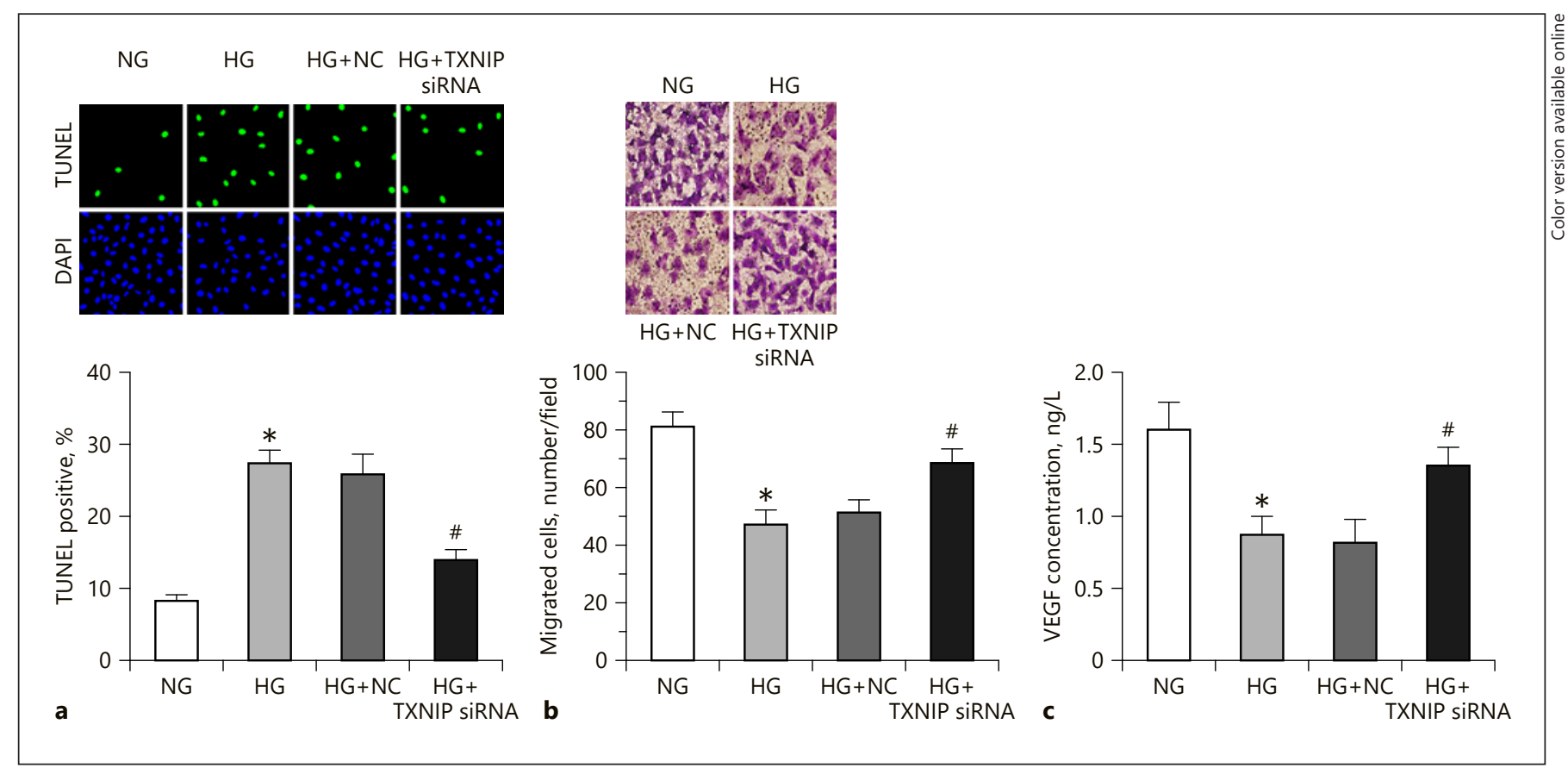

Fig. 5. High glucose mediated the impaired angiogenic abilities in endothelial cells via upregulation of TXNIP $(n=5, * p<0.05$ vs. $\mathrm{NG},{ }^{\#} p<0.05$ vs. HG + NC). a Apoptosis in endothelial cells measured by TUNEL assay. Representative images are shown in the upper panel. Quantitative analysis is indicated in the lower panel.

To establish the causative relationship, TXNIP gene expression was silenced in HUVECs using siRNA under high glucose condition. As a result, angiogenic abilities of endothelial cells were improved, evidenced by decreased apoptosis, enhanced migration, and elevated VEGF secretion (Fig. 5). These results suggest that high glucoseinduced Trx1 inactivation due to TXNIP upregulation mediated the impaired angiogenic abilities in endothelial cells.

\section{TXNIP Knockdown or Recombinant Human \\ Trx1 Treatment Counteracted the Impairment of Angiogenesis and Alleviated Myocardial Ischemic Injury in Diabetic Mice}

Based on the data above, we hypothesize that Trx1 inactivation affects ischemia-induced angiogenesis in diabetic myocardium. To further test this hypothesis, knockdown of TXNIP or supplementation with exogenous Trx1 was performed in diabetic MI model. As shown in Figure 6, recombinant human Trx1 supplementation or pretreatment with shTXNIP increased endothelial cell proliferation activity and capillary density in diabetic myocardium after MI, indicated by increased percentage b Migration in endothelial cells measured by Transwell chamber and crystal violet staining. Representative images are shown in the upper panel. Quantitative analysis by counting stained cells is indicated in the lower panel. c VEGF concentrations in medium measured by ELISA kits.

of Ki67 and CD31 double positive cells. Consistent with the angiogenesis restoration, cardiac systolic function and survival rate were improved to the levels comparable to those in the control MI group (Fig. 6). All the above data indicate that upregulation of TXNIP with subsequent inhibition of Trx1 activity plays a critical role in DM-induced impairment of angiogenesis and intensified injury after MI.

\section{Discussion}

Angiogenesis, the formation of new blood vessels from preexisting ones, contributes to a vast array of physiological and pathological conditions. Abnormalities of angiogenesis cause many clinical manifestations of diabetes. A complicated feature of the abnormal angiogenesis is that insufficient and excessive angiogenesis can occur in diverse organs in the same individual [2]. For the heart, angiogenesis is a key compensatory mechanism in response to the ischemia created by coronary artery disease. MI undoubtedly will trigger a spontaneous protective angiogenic response, which aims at reestablishing 


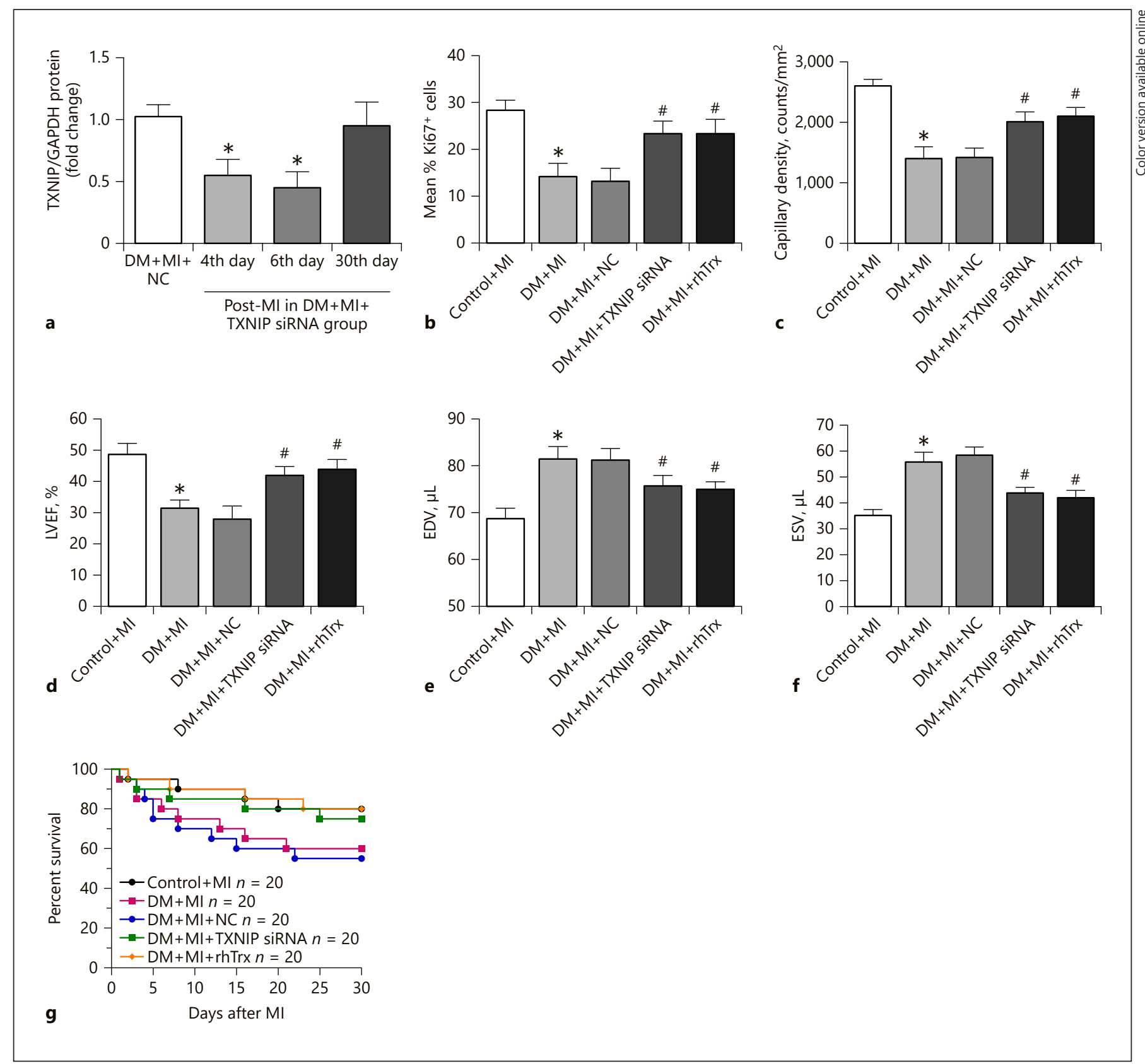

Fig. 6. TXNIP knockdown or recombinant human Trx1 (rhTrx1) treatment counteracted the impairment of angiogenesis and alleviated myocardial ischemic injury in diabetic mice $\left(n=6,{ }^{*} p<0.05\right.$ vs. control $+\mathrm{MI},{ }^{*} p<0.05$ vs. DM $\left.+\mathrm{MI}+\mathrm{NC}\right)$. a TXNIP protein expression in a time course in mice silenced with TXNIP. b Percentage of Ki67 and CD31 double positive cells determined by

blood flow, reducing myocardial injury, and preventing the adverse remodeling. Unfortunately, diabetes-induced decline of collateral vessel growth in ischemic myocardium has been shown by several studies $[1,2]$, immunofluorescence staining. c Quantitative analysis of vessel density determined by immunohistochemistry staining of CD31. $\mathbf{d}-\mathbf{f}$ Left ventricular ejection fraction (LVEF), EDV, and ESV values determined by echocardiography. $\mathbf{g}$ Survival rates indicated in survival curve. 
by the fact that promoting angiogenesis improved myocardial function and remodeling. Nevertheless, the underlying mechanism remains unclear [13]. In this study, we first demonstrated that the impairment of a balanced status of TXNIP-Trx1 system plays a vital role in the process.

Trx is a multifunctional protein involved in the regulation of cellular homeostasis. Accumulating evidence supports the notion that Trx might represent a promising therapeutic target for the prevention and treatment of cardiovascular disease $[3,4]$. We have previously reported that Trx 1 inactivation increased vulnerability of diabetic hearts to I/R injury $[5,6]$. Trx system was also implicated to play a role in endothelial cell homeostasis and key angiogenic process, including endothelial cell migration, proliferation, and survival. Endothelial-specific expression of Trx promotes ischemia-mediated angiogenesis in ischemic limb [14]. Trx1 gene upregulation enhanced angiogenic signaling and reduced ventricular remodeling in infarcted myocardium of rodents $[8,9]$. However, the status of endogenous Trx system in diabetic individuals and whether it mediates the impaired angiogenesis in ischemic myocardium is still to be identified. In the present study, we found that Trx 1 inactivation mediated the impaired angiogenic abilities in endothelial cells under high glucose condition, and decreased angiogenesis in infarcted diabetic myocardium.

TXNIP has been of great interest in the past decade since it has been reported to be a critical regulator in human diseases, especially diabetes [15]. The promoter of TXNIP contains a carbohydrate response element responsible for the hyperglycemia-induced TXNIP expression. To date, TXNIP expression has been found to be elevated in all diabetic tissues assessed, contributing to the injury in various types of cells [7]. High glucose levels induced TXNIP expression in rat cardiomyocytes, which was responsible for impaired cardiac inotropic reserve in diabetic myocardium [16]. TXNIP, initially identified as an endogenous regulator of Trx, inhibits Trx1 activity by forming a mixed disulfide bond and blocks Trx1-dependent gene transcription and expression, influencing cell activity. In vitro, TXNIP repression increased Trx activity and strongly induced migration in endothelial cells [17]. But the role of Trx1 and TXNIP interaction in MI-induced angiogenesis in diabetic myocardium has not been understood. Under this condition, our data indicated that Trx1 inactivationmediated poor angiogenesis is due to the increase of TXNIP expression and TXNIP-Trx1 complex in endo- thelial cells. Su et al. [18] found that hyperglycemia enhances TXNIP expression in cardiomyocytes, leading to aggravated cardiac injury following I/R. Recently, Kim et al. [19] also reported that in acute hyperglycemic state, melatonin treatment effectively ameliorated myocardial I/R injury by downregulating TXNIP expression and rescuing intracellular Trx system in cardiomyoblasts. Together with our results, all suggest a vital role of TXNIP-Trx 1 axis in diabetes-intensified myocardial ischemia injury. Similarly, TXNIP knockdown to nondiabetic levels was shown to rescue diabetes-related impairment of angiogenesis, blood flow, and functional recovery in ischemic hindlimb [20]. Interestingly, contrary to our data, in diabetic model of murine hindlimb ischemia, the effect of TXNIP on angiogenesis is independent of TXNIP's ability to bind to Trx and modulating the activity of $\operatorname{Trx}[20]$. The mechanisms underlying the different effects of TXNIP upregulation in different tissues of diabetic subjects are not fully understood. Capillary density and reactions of neighbor cells to the hypoxia may all involve in the pathological process. In our previous study, TXNIP mediated insufficient angiogenesis also via impaired $\mathrm{Wnt} / \beta$-catenin signaling in diabetic myocardium after MI [21]. Thus, in endothelial cells, Trx-dependent and Trx-independent mechanisms may both exist underlying the antiangiogenic action caused by TXNIP expression.

In summary, the results of the current study strongly suggest that TXNIP upregulation and subsequently the increased formation of TXNIP-Trx1 complex is a novel pathologic pathway by which diabetes induces impairment of angiogenesis and thereby exacerbates myocardial ischemia injury. Blocking TXNIP overexpression or supplementation of exogenous Trx1 significantly reversed the impairment of angiogenesis, alleviated myocardial injury, and improved survival rate. These results imply that TXNIP-Trx1 system might represent a promising therapeutic target for improving diabetic patient outcomes after MI injury.

\section{Acknowledgements}

This study was supported by the following grants: National Natural Science Foundation of China (NSFC.81300078, NSFC.81500202, NSFC.81870185). China Postdoctoral Science Foundation (CPSF, 2015M582808, 2016T90993). Hainan science and technology project (ZDKJ2016008, ZDYF2017096, ZDYF2018118). Open subject of National Clinical Research Center of Geriatrics Disease (NCRCG-PLAGH-2018014).
Hou/Shen/Wang/Liu/Gao/Xu/Tao/Yin/ Yin 


\section{Statement of Ethics}

All experiments involving animals were performed in adherence with the Guide for the Care and Use of Laboratory Animals published by the US National Institutes of Health (NIH Publication No. 85-23, revised 1996), and approved by the Fourth Military Medical University Committee on Animal Care.

\section{Disclosure Statement}

The authors have no conflicts of interest to disclose.

\section{Author Contributions}

Z.Y. and T.Y. designed the studies, R.H., M.S., R.W., H.L., and C.G. conducted the studies, J.X. and L.T. analyzed the data, R.H., Z.Y., and T.Y. wrote the manuscript. All authors read and approved the final manuscript.

\section{References}

1 Mouquet F, Cuilleret F, Susen S, Sautière K, Marboeuf P, Ennezat PV, et al. Metabolic syndrome and collateral vessel formation in patients with documented occluded coronary arteries: association with hyperglycaemia, insulin-resistance, adiponectin and plasminogen activator inhibitor-1. Eur Heart J. 2009 Apr;30(7):840-9.

2 Kota SK, Meher LK, Jammula S, Kota SK, Krishna SV, Modi KD. Aberrant angiogenesis: the gateway to diabetic complications. Indian J Endocrinol Metab. 2012 Nov;16(6): 918-30.

3 Yamawaki H, Haendeler J, Berk BC. Thioredoxin: a key regulator of cardiovascular homeostasis. Circ Res. 2003 Nov;93(11):102933.

4 Whayne TF Jr, Parinandi N, Maulik N. Thioredoxins in cardiovascular disease. Can J Physiol Pharmacol. 2015 Nov;93(11):903-11.

5 Luan R, Liu S, Yin T, Lau WB, Wang Q, Guo $\mathrm{W}$, et al. High glucose sensitizes adult cardiomyocytes to ischaemia/reperfusion injury through nitrative thioredoxin inactivation. Cardiovasc Res. 2009 Jul;83(2):294-302.

6 Yin T, Hou R, Liu S, Lau WB, Wang H, Tao L. Nitrative inactivation of thioredoxin-1 increases vulnerability of diabetic hearts to ischemia/reperfusion injury. J Mol Cell Cardiol. 2010 Sep;49(3):354-61.

7 Dunn LL, Buckle AM, Cooke JP, Ng MK. The emerging role of the thioredoxin system in angiogenesis. Arterioscler Thromb Vasc Biol. 2010 Nov;30(11):2089-98.

8 Samuel SM, Thirunavukkarasu M, Penumathsa SV, Koneru S, Zhan L, Maulik G, et al. Thioredoxin-1 gene therapy enhances angio- genic signaling and reduces ventricular remodeling in infarcted myocardium of diabetic rats. Circulation. 2010 Mar;121(10):124455.

9 Adluri RS, Thirunavukkarasu M, Zhan L, Akita Y, Samuel SM, Otani H, et al. Thioredoxin 1 enhances neovascularization and reduces ventricular remodeling during chronic myocardial infarction: a study using thioredoxin 1 transgenic mice. J Mol Cell Cardiol. 2011 Jan;50(1):239-47.

10 Kaga S, Zhan L, Matsumoto M, Maulik N. Resveratrol enhances neovascularization in the infarcted rat myocardium through the induction of thioredoxin-1, heme oxygenase-1 and vascular endothelial growth factor. J Mol Cell Cardiol. 2005 Nov;39(5):813-22.

11 Li X, Kover KL, Heruth DP, Watkins DJ, Moore WV, Jackson K, et al. New Insight Into Metformin Action: Regulation of ChREBP and FOXO1 Activities in Endothelial Cells. Mol Endocrinol. 2015 Aug;29(8): 1184-94.

12 Gao E, Lei YH, Shang X, Huang ZM, Zuo L, Boucher M, et al. A novel and efficient model of coronary artery ligation and myocardial infarction in the mouse. Circ Res. 2010 Dec; 107(12):1445-53.

13 Shi Y, Vanhoutte PM. Macro- and microvascular endothelial dysfunction in diabetes. Diabetes. 2017 May;9(5):434-49.

14 Dai S, He Y, Zhang H, Yu L, Wan T, Xu Z, et al. Endothelial-specific expression of mitochondrial thioredoxin promotes ischemiamediated arteriogenesis and angiogenesis. Arterioscler Thromb Vasc Biol. 2009 Apr; 29(4):495-502.
15 Schroder K, Zhou R, Tschopp J. The NLRP3 inflammasome: a sensor for metabolic danger? Science. 2010 Jan;327(5963):296-300.

16 Myers RB, Fomovsky GM, Lee S, Tan M, Wang BF, Patwari P, et al. Deletion of thioredoxin-interacting protein improves cardiac inotropic reserve in the streptozotocin-induced diabetic heart. Am J Physiol Heart Circ Physiol. 2016 Jun;310(11):H1748-59.

17 Ng MK, Wu J, Chang E, Wang BY, Katzenberg-Clark R, Ishii-Watabe A, et al. A central role for nicotinic cholinergic regulation of growth factor-induced endothelial cell migration. Arterioscler Thromb Vasc Biol. 2007 Jan;27(1):106-12.

18 Su H, Ji L, Xing W, Zhang W, Zhou H, Qian $\mathrm{X}$, et al. Acute hyperglycaemia enhances oxidative stress and aggravates myocardial ischaemia/reperfusion injury: role of thioredoxin-interacting protein. J Cell Mol Med. 2013 Jan;17(1):181-91.

19 Kim GS, Kim BK, Shin DH, Kim JS, Hong MK, Gwon HC, et al.; K-CTO Registry. Predictors of poor clinical outcomes after successful chronic total occlusion intervention with drug-eluting stents. Coron Artery Dis. 2017 Aug;28(5):381-6.

20 Dunn LL, Simpson PJ, Prosser HC, Lecce L, Yuen GS, Buckle A, et al. A critical role for thioredoxin-interacting protein in diabetesrelated impairment of angiogenesis. Diabetes. $2014 \mathrm{Feb} ; 63(2): 675-87$.

21 Shen M, Bai D, Liu B, Lu X, Hou R, Zeng C, et al. Dysregulated Txnip-ROS-Wnt axis contributes to the impaired ischemic heart repair in diabetic mice. Biochim Biophys Acta Mol Basis Dis. 2018 Dec;1864(12):3735-45. 